\title{
Asymmetric poly(ethylene-alt-propylene)-poly(ethylene oxide) micelles: A system with starlike morphology and interactions
}

\author{
M. Laurati, ${ }^{1, *}$ J. Stellbrink, ${ }^{1}$ R. Lund, ${ }^{1, \dagger}$ L. Willner, ${ }^{1}$ E. Zaccarelli, ${ }^{2}$ and D. Richter ${ }^{1}$ \\ ${ }^{1}$ Institut für Festkörperforschung, Forschungszentrum Jülich, 52425 Jülich, Germany \\ ${ }^{2}$ Dipartimento di Fisica and INFM-CRS SOFT, Università di Roma La Sapienza, Piazza A. Moro 2, I-00185, Roma, Italy
}

(Received 4 May 2007; published 18 October 2007)

\begin{abstract}
We report on an experimental study of single particle properties and interactions of poly(ethylene-altpropylene)-poly(ethylene oxide) (PEP-PEO) starlike micelles. The starlike regime is achieved by an extremely asymmetric block ratio (1:20) and the number of arms (functionality) is changed by varying the composition of the solvent (the interfacial tension). Small angle neutron scattering (SANS) data in the dilute regime can be modeled by assuming a constant density profile in the micellar core (compact core) and a starlike density profile in the corona (starlike shell). The starlike morphology of the corona is confirmed by a direct comparison with SANS measurements of dilute poly butadiene star solutions. Comparison of structure factors obtained by SANS measurements in the concentrated regime shows in addition that the interactions in the two systems are equivalent. Micellar structure factors at several packing fractions can be modeled by using the ultrasoft potential recently proposed for star polymers [Likos et al., Phys. Rev. Lett. 80, 4450 (1998)]. The experimental phase diagram of PEP-PEO micelles is quantitatively compared to theoretical expectations, finding good agreement for the location of the liquid-solid boundary and excellent agreement for the critical packing fraction where the liquid-to-bcc crystal transition takes place for $f<70$. The functionality, i.e., the coronal density, strongly influences the nature of the solid phase: for $f<70$ the system crystallizes into a bcc phase, high $f$ $>70$ formation of amorphous arrested states prevents crystallization.
\end{abstract}

DOI: 10.1103/PhysRevE.76.041503

PACS number(s): 64.70.Nd, 61.12.Ex, 61.25.Hq, 82.35.Jk

\section{INTRODUCTION}

Star polymers constitute a natural bridge between linear polymer chains and hard sphere colloids [2]. Their softness is controlled by the functionality $f$, i.e., the number of polymer chains tethered to the central microscopic core. For $f=2$ the morphology of the star is that of a linear polymer chain while for very large $f$ it resembles a spherical colloidal particle. This adjustable softness affects morphology as well as starstar interactions. The effective interaction potential introduced by Likos et al. [1] presents a logarithmic dependence of the repulsion between two stars as a function of the center-to-center distance $(r)$, for values of $r$ smaller than the particle diameter. The strength of the repulsion is increasing with $f$ and in the limit of $f \rightarrow \infty$ the hard sphere potential is recovered. Such a potential was shown to give a correct description of small angle neutron scattering (SANS) data of star polymer solutions $[1,3]$, while the hard-sphere interaction potential failed [4]. Starting from the potential of Ref. [1], the phase diagram of star polymer solutions was calculated, as a function of $f$ and packing fraction $\eta$ [5]. Due to the unusual soft repulsion between stars, a variety of equilibrium crystalline phases were predicted depending on $\eta$ and $f$. Recently the occurrence of nonequilibrium states was also taken into account. The mode-coupling theory (MCT) ideal glass transition line was found to closely track the

\footnotetext{
*Present address: Lehrstuhl für Physik der Weichen Materie, IPkM, Heinrich-Heine Universität Düsseldorf, 40225 Düsseldorf, Germany; marco.laurati@uni-duesseldorf.de

†Present address: Donostia International Physics Center, Paseo Manuel de Lardizabal 4, 20018 San Sebastián, Spain.
}

fluid-solid boundary in the equilibrium phase diagram [6].

Experimental investigation of star polymer solutions as a function of concentration and functionality $f$ was qualitatively confirming some of the features predicted by theory $[3,4,7]$, e.g., the formation of solid phases with increasing polymer volume fraction $\phi$. Concerning the nature of the solid phases observed at high volume fractions, there was little evidence of crystalline phases [body-centered-cubic (bcc) [8] and face-centered-cubic (fcc) [8,9] at large $f$ values], while in many cases the system was found to be trapped in a metastable arrested state $[3,7,10]$. However, a systematic investigation of the experimental phase diagram of star polymers and a quantitative comparison with theory is still missing.

One of the main problems is that the high interest in star polymers is not reflected in their availability. Synthesis requires considerable preparative efforts and due to the dendrimer nature of the star core, the functionality $f$ cannot be smoothly adjusted [2]. It would then be preferable to use a readily available model system for star polymers that should not only show the typical molecular architecture of a star, but also interact via the effective potential proposed for star polymers. A natural candidate for such a model system is diblock copolymer micelles. A major task in a micellar system is that of approaching the starlike regime $[11,12]$ defined by the block ratio $N_{a} / N_{b} \gg 1$, with $N_{a} / N_{b}$ the degrees of polymerization of the soluble-insoluble block. In fact very few block copolymers with asymmetric composition selfassemble into micellar structures since an extreme incompatibility between the blocks and between insoluble block and solvent is required. Two micellar systems turned out to be ideal in this respect: hydrophobically modified poly(ethylene oxide) PEO [13-15] in water and asymmetric poly(ethylene- 
alt-propylene)-poly(ethylene oxide) (PEP-PEO) block copolymers in water and water/N,N-dimethylformamide (DMF) mixtures $[16,17]$. These systems form micelles with small micellar cores and large extended coronas typical for starlike morphologies. The aggregation numbers are in the range between 30 and 130 ideally matching the functionality of regular star polymers accessible by living anionic polymerization.

With regard to interactions, a few block copolymer micellar systems have been related to star polymers, even though in all cases the starlike regime was not achieved. In their pioneering work, Gast et al. investigated the dependence on block composition $\left(0.8 \leq N_{a} / N_{b} \leq 4\right)$ of the lattice geometry (fcc or bcc) of crystalline phases of poly(styrene)poly(isoprene) (PS-PI) block copolymers [18]. They found that increasing block ratio, i.e., increasing the repulsion range, the bcc crystalline phase is stabilized against the fcc phase, in analogy to what is predicted for a reduction of the star functionality $f$. More recently bcc and fcc phases were observed in PS-PI [19,20] and poly(ethylene oxide)poly(butylene oxide) (PEO-PBO) [21] micelles. In both cases, temperature was used as a control parameter for the transition between these two crystalline lattices. In Ref. [20] temperature changes were shown to induce variation of the aggregation number $f$ and the fcc-bcc transition was compared to the phase diagram of star polymers, indicating some similarities in the interactions. However, the block ratio was rather small, $N_{a} / N_{b} \approx 2[20]$.

In the present study we utilize PEP-PEO micelles prepared from block copolymers of very asymmetric composition $\left(N_{a} / N_{b} \approx 20\right)$ in aqueous solution as a star model system. Single particle properties and interactions were systematically investigated by SANS covering a broad concentration range. The results are successfully compared to theoretical predictions for regular star polymers. The starlike nature of the system is confirmed by a direct comparison to experimental SANS data for polybutadiene (PB) star polymer solutions in the dilute and concentrated regime. The functionality $f$ of PEP-PEO can be effectively tuned by the addition of DMF allowing one to study the star polymers phase diagram over a broad range of functionalities $f$ and volume fractions $\phi$.

The results for single particle properties will be presented first in Sec. III A. The SANS measurements were performed at low concentrations. The PEO-block has been selectively deuterated, such that the micellar structure could be studied by individual contrasts (core contrast, shell contrast). This allows an accurate determination of micellar functionalities (core contrast) and verification of the starlike morphology of the micelles (shell contrast).

Subsequently, in Sec. III B, on the basis of experimental structure factors obtained from SANS measurements, we show the comparison between interactions of PEP-PEO micelles and of PB real star polymers. In Sec. III C the experimental phase diagram of PEP-PEO micelles is presented and discussed in terms of the theoretical phase diagram of star polymer solutions. Quantitative comparison to theory was obtained by modeling experimental structure factors $S(Q)$, starting from the ultrasoft potential of Ref. [1]. The parameters controlling the interaction, $\phi, f$, and the interaction
TABLE I. Characterization of PEP-PEO block copolymer. $D_{p}$ is the degree of polymerization, $x_{h}$ the mole fraction of $h$-monomer, and $\rho$ the scattering length densities of polymer blocks.

\begin{tabular}{lrcrcc}
\hline \hline & \multicolumn{1}{c}{$M_{n}$} & $M_{W} / M_{n}$ & $D_{p}$ & $x_{h}$ & $\rho\left[10^{10} \mathrm{~cm}^{-2}\right]$ \\
\hline PEP (block) & 1100 & 1.06 & 15 & 1.0 & -0.31 \\
PEO (block) & 20700 & $1.04^{\mathrm{a}}$ & 436 & 0.11 & 6.32 \\
\hline \hline
\end{tabular}

${ }^{a}$ Overall polydispersity by SEC.

length $\sigma$ were directly given by experimental values. We illustrate how experimental data for PEP-PEO micelles reproduce to a high level of accuracy the liquid-solid transition of the theoretical phase diagram (Sec. III C). Moreover, in agreement with theory, we show that $f$ and thus the chain density of the micellar corona strongly influences the possibility for the system to crystallize. Concerning crystalline phases, we show that on the basis of simple arguments a heterogeneous crystalline structure is expected. Part of the results shown here have been presented in a recent paper [22].

\section{EXPERIMENTAL METHODS}

\section{A. Micellar properties and sample preparation}

The micellization behavior of PEP-PEO in aqueous solution is governed by the large interfacial tension, $\gamma$ $=46 \mathrm{mN} / \mathrm{m}$, between PEP and water. Such behavior was thoroughly studied by us during the last years [16,23], resulting in the following findings. (i) PEP-PEO forms micelles with the hydrophobic PEP constituting the core and the hydrophilic PEO constituting the corona. (ii) Micelles are even formed in a very asymmetric block composition with high PEO content $\left(N_{a} / N_{b} \approx 20\right)$. (iii) All micellar cores are completely segregated, i.e., not swollen by solvent. (iv) The micelles are kinetically frozen in water: Although chemically not linked, exchange of block copolymers between different micelles could not be observed, not even at elevated temperatures [24-26]. This means that no free chains, i.e., no depletion effects [7] are present. (v) Adjusting $\gamma$ by addition of a PEO selective cosolvent like N,N-dimethylformamide (DMF) allows one to smoothly vary $f$ even in the starlike regime (where $f \sim \gamma^{6 / 5}$ was confirmed by experiments [17]). Recent measurements of exchange kinetics in water/DMF mixtures $[25,26]$ observed significant block copolymers exchange at a DMF mole fraction $\left(x_{D M F}\right)$ larger than 0.4. The relatively high interfacial tension measured at $x_{D M F}=0.4$ [24-26], $\gamma=16.5 \mathrm{mN} / \mathrm{m}$, indicates that despite the significant block copolymer exchange, the amount of free chains in solution should not induce significant depletion effects.

The asymmetric PEP-PEO block copolymer under study was synthesized by anionic polymerization [23]. To exploit $h / d$-contrast variation in our SANS experiments, the individual blocks have been selectively protonated-deuterated, see Table I. The partial deuteration of the PEO chain was obtained by premixing deuterated and protonated EO monomers prior to the polymerization, leading to a copolymer with a random distribution of $h$ - and $d$-repeat units. The deu- 
teration degree was calculated from the initial amounts of $h$ and $d$ materials. The block copolymer and related PEP-OH parent materials were characterized by a combination of size exclusion cromatography (SEC), ${ }^{1} \mathrm{H}-\mathrm{NMR}$, and end group analysis [17,23].

Scattering length densities of the single blocks were calculated according to

$$
\rho_{i}=\frac{\sum_{j} b_{j}}{v_{i}},
$$

with $i$ indicating the PEP or PEO monomer, $\Sigma_{j} b_{j}$ the sum of the coherent scattering lengths of all atoms in monomer $i$, and $v_{i}$ the average volume of one molecule of monomer $i$ :

$$
v_{i}=\frac{M_{i}}{\delta_{i} N_{A}}
$$

with $M_{i}$ the molar mass of component $i$ and $\delta_{i}$ the corresponding density.

The density of the PEP block was taken from [24], where it was measured by means of an Anton Paar DMA 5000 density meter. The density $\delta$ of the PEO block was estimated using relation $\delta_{h d}^{P E O}=0.89 \delta_{d}^{P E O}+0.11 \delta_{h}^{P E O}$, where the bulk density of $h$-PEO $\left(\delta_{h}^{P E O}\right)$ was taken from [27] and the density of $d$-PEO $\left(\delta_{d}^{P E O}\right)$ was calculated from that of the hydrogenated material by assuming identical volume.

Using water/DMF mixtures we prepared samples with six different $f$ going from dilute $\left(0.25 \times 10^{-2}\right.$ polymer volume fraction) to the very concentrated regime (up to $\approx 0.27$ polymer volume fraction). Binary mixtures of $\mathrm{H}_{2} \mathrm{O} / \mathrm{D}_{2} \mathrm{O}$ and quaternary mixtures of $\mathrm{H}_{2} \mathrm{O} / \mathrm{D}_{2} \mathrm{O} / \mathrm{DMF} / d$-DMF with different volume fractions of the components have been prepared for different contrast conditions. Water/DMF mixtures were found to be nonideal [17]. Therefore the average densities of the $\mathrm{H}_{2} \mathrm{O} / \mathrm{DMF}$ and $\mathrm{D}_{2} \mathrm{O} / d$-DMF mixtures were separately measured with an Anton Paar DMA 5000 density meter. The average scattering length densities of the hydrogenated $\left(\rho_{h-\text { mixt }}\right)$ and deuterated $\left(\rho_{d-\text { mixt }}\right)$ mixtures were calculated as described in [17]. Finally the scattering length density of the quaternary mixtures $\left(\rho_{0}\right)$ were calculated using the additive relation $\rho_{0}=\phi_{h-\text { mixt }} \rho_{h-\text { mixt }}+\phi_{d-\text { mixt }} \rho_{d-\text { mixt }}$. Values of $\rho_{0}$ for the different solvent mixtures are listed in Table II.

The preparation of dilute solutions was already described in detail elsewhere [17]. Concentrated solutions were prepared by mixing weighted amounts of solvent and polymer. Solvents were filtrated two times through Anotop membrane filters of $20 \mathrm{~nm}$ pore size. Samples were prepared separately directly in the measurement cells. The stability of the aggregation number of the micelles in the different samples was crosschecked in the SANS measurements, as will be shown later in the paper (Sec. II B 1). To homogenize the samples magnetic stirring balls of 0.5 and $1 \mathrm{~mm}$ diameter were introduced inside the cells and moved by applying an external weak magnetic field. After homogenization, samples were left at rest for at least $12 \mathrm{~h}$ (a few days in most of the cases) in order to equilibrate.
TABLE II. Characterization of PEP-PEO micelles. $x_{D M F}$ is the mole fraction of DMF in the solvent and $\rho_{0}$ is the scattering length density of the solvent. $R_{c, \min }$ and $f_{\min }$ are the core radius and functionality from minimum position, $R_{c, f i t}$ and $f_{f i t}$ are the core radius and functionality from fit to the compact core model, and $R_{m}$ is the micellar radius obtained from fit to the core-shell model.

\begin{tabular}{lccrccc}
\hline \hline & $\begin{array}{c}\rho_{0} \\
x_{D M F}\end{array}$ & $\begin{array}{c}R_{c, \min } \\
\left(10^{10} \mathrm{~cm}^{-2}\right)\end{array}$ & $\begin{array}{c}R_{c, f i t} \\
(\AA)\end{array}$ & $f_{\text {min }}$ & $\begin{array}{c}R_{m} \\
(\AA)\end{array}$ \\
\hline 0.0 & 6.33 & 42 & 136 & $42 \pm 1$ & $140 \pm 10$ & $288 \pm 12$ \\
0.1 & 6.32 & 37 & 94 & $36.4 \pm 0.5$ & $91 \pm 4$ & $265 \pm 13$ \\
0.2 & 6.34 & 35 & 82 & $35.1 \pm 0.5$ & $83 \pm 3.5$ & $243 \pm 12$ \\
0.3 & 6.33 & 34 & 73 & $33.8 \pm 0.4$ & $74 \pm 3$ & $222 \pm 10$ \\
0.4 & 6.31 & 33 & 67 & $32.8 \pm 0.5$ & $68 \pm 3$ & $208 \pm 6$ \\
0.5 & 6.31 & 32 & 63 & $32.5 \pm 0.4$ & $66 \pm 2$ & $200 \pm 7$ \\
\hline \hline
\end{tabular}

\section{B. SANS experiments}

\section{Measurements}

Small angle neutron scattering experiments have been performed at KWS-1 and KWS- 2 instruments (Forschungszentrum Jülich), at PAXY (Laboratoire Leon Brillouin), at D11 (Institute Laue Langevin), and at SANS-I (Paul Scherrer Institut). Details about different setups and instrument specifications can be found in [28]. SANS data were corrected following standard procedures and normalized to absolute units to allow a quantitative theoretical interpretation (see, for example, [29]).

The macroscopic scattering cross section, $(d \Sigma / d \Omega)(Q)$, measured by SANS can be expressed, for monodisperse particles with spherical symmetry, as a product of single particle contributions, the particle form factor $P(Q)$, and the structure factor $S(Q)$, which contains all information about particle interactions:

$$
\left(\frac{d \Sigma}{d \Omega}\right)(Q)=N_{z} P(Q) S(Q)
$$

$Q=(4 \pi / \lambda) \sin (\theta / 2)$ is the modulus of the scattering vector $\mathbf{Q}$, defined as $\mathbf{Q}=\mathbf{k}_{i}-\mathbf{k}_{s}$ where $\mathbf{k}_{i}$ is the wave vector of the incoming neutrons and $\mathbf{k}_{s}$ is the wave vector of the scattered neutrons. $\lambda$ is the neutron wavelength and $\theta$ is the scattering angle. Here $N_{z}$ is the number density of particles. In the following we will refer to the scattering cross section as SANS intensity and we will use the nomenclature $I(Q)$.

We measured particle form factors in dilute solution for all different solvent mixtures. Adjusting the scattering length density $\rho_{0}$ of the solvent by use of $h / d$-isotopic mixtures to that of the PEO/PEP reduces the contrast factor between corona/core and solvent to zero, see Table II. Therefore core form factors and corona form factors were independently measured.

Experimental $S(Q)$ can be extracted from SANS data measured in concentrated solutions by dividing out the experimental $P(Q)$ measured in dilute solution. However, this procedure is only valid if particle size and shape, i.e., $P(Q)$, are unaffected by concentration. This is in general not the 
case for deformable particles like micelles. Moreover, following the approach of Kotlarchyk and Chen for polydisperse particles [30], it has been shown in recent studies that for block copolymer micelles the factorization of the scattering intensity into a form and structure factor following Eq. (3) in general does not hold due to lack of spherical symmetry of the micellar corona [31]; but we can overcome these problems by performing SANS experiments for concentrated solutions in core contrast only. Indeed the PEP core is compact, spherically symmetric, and completely unaffected by increasing concentration. The validity of this assumption can be rationalized from Fig. 6, where the minimum arising from the solid core appears, at each fixed value of $f$, at the same $Q$ value (within error bars) independent of $\phi$, i.e., the core form factor is unchanged with increasing $\phi$.

\section{Theoretical models}

Form factor. During the last years several different models for describing form factors of diblock copolymer micelles have been proposed. A recent review of the different models can be found in [32,33]. A very accurate model has been recently proposed by Svaneborg and Pedersen, where shortrange correlations and excluded volume interactions between chains in the corona have been quantitatively taken into account $[31,34]$. In the brush regime, the block copolymer micelles can be alternatively modeled as a core-shell structure [35], with a homogeneous density profile in the core and a starlike density profile in the shell. The scattering contribution of the corona chains, "blob scattering," has to be implemented as an additional term using the Beaucage function [36]. Such an empirical model has been successfully used in previous studies to describe scattering data from PEP-PEO micelles in water and water/DMF mixtures $[16,17]$.

The scattering intensity measured in the dilute regime, in a generic contrast, was modeled according to the following expression:

$$
I(Q)=N_{z} P(Q)+I_{\text {blob }}(Q),
$$

where $I_{b l o b}(Q)$ is the contribution due to the correlations between the chains in the micellar corona, the so-called blob scattering, see Eq. (11). The micellar form factor $P(Q)$ was expressed as the ensemble average of the squared scattering amplitude $A(Q)$ :

$$
P(Q)=\left\langle|A(Q)|^{2}\right\rangle .
$$

The scattering amplitude of micelles formed by a compact PEP core and a diffuse PEO corona was described using a spherical core-shell model, according to the following relation $[16,23]$ :

$$
A(Q)=\left(\rho_{c}-\rho_{0}\right) V_{c} A(Q)_{c}+\left(\rho_{s h}-\rho_{0}\right) V_{s h} A(Q)_{s h},
$$

where $V_{c}$ and $V_{s h}$ are the core volume and the corona volume, respectively. $A(Q)_{c}$ and $A(Q)_{s h}$ denote the core and shell scattering amplitudes. The partial scattering amplitudes of core and shell in spherical symmetry can be written as the Fourier transform of the radial density distribution, $n(r)[16]$ :

$$
A(Q)_{c, s h}=\frac{1}{C} \int_{0}^{\infty} 4 \pi r^{2} n(r) \frac{\sin (Q r)}{Q r} d r,
$$

with $C=\int_{0}^{\infty} n(r) 4 \pi r^{2} d r$.

The radial density distribution in the core was assumed to be constant. The possible presence of surface roughness or a diffuse interface between core and corona were taken into account by convoluting the density distribution with a Gaussian distribution function, leading to

$$
A(Q)_{c}=\frac{3 \sin \left(Q R_{c}\right)-Q R_{c} \cos \left(Q R_{c}\right)}{\left(Q R_{c}\right)^{2}} \exp \left[-\left(\sigma_{c} Q\right)^{2} / 4\right],
$$

where $\sigma_{c}$ is the smearing parameter. Here $R_{c}$ is the core radius, given by

$$
R_{c}=\left(\frac{3 f V_{c}}{4 \pi}\right)^{1 / 3} .
$$

It was assumed a compact, solvent, and PEO free core with a total volume $V_{c}=f V_{P E P}$, where $V_{P E P}$ is the volume of a PEP chain.

The shell was described using a hyperbolic distribution, combined with a Fermi function in order to have a cutoff of the power law dependence, according to the finite size of the polymer chain [16].

$$
n(r)=\frac{r^{-4 / 3}}{1+\exp \left[\left(r-R_{m}\right) /\left(\sigma_{m} R_{m}\right)\right]},
$$

with $R_{m}$ the overall micellar radius and $\sigma_{m}$ the smearing parameter that adjusts the cutoff distance of the Fermi function. The value $4 / 3$ for the exponent of the hyperbolic function is typical for starlike structures [16].

As anticipated, in order to describe blob scattering contributions to the scattering cross section we used the Beaucage function [36]:

$$
I_{\text {blob }}(Q)=\left(\rho_{\text {sh }}-\rho_{0}\right)^{2} B\left(\frac{[\operatorname{erf}(Q \xi / \sqrt{6})]^{3}}{Q}\right)^{D},
$$

where erf is the error function, $B$ is an intensity prefactor proportional to the blob volume, $\xi$ is the blob radius, and $D$ the fractal dimension of the object.

Structure factor. Pair correlation functions $g(r)$, where $r$ is the distance between the centers of two particles, were calculated by solving the Ornstein-Zernike integral equation with the Rogers-Young (RY) closure relation [37]:

$$
g(r)=\exp [-\beta v(r)]\left[1+\frac{\exp \{f(r)[h(r)-c(r)]-1\}}{f(r)}\right],
$$

where $\beta=1 / k_{B} T, v(r)$ is the pair interaction potential, $h(r)$ $=g(r)-1$ is the total correlation function, $c(r)$ is the direct correlation function, and $f(r)=1-\exp (-\alpha r)$. The parameter $\alpha$ is used to achieve thermodynamic consistency. The RY closure relation was shown to give satisfactory agreement with simulations for many repulsive types of interactions, including star polymers [38,39], star polymer mixtures 
[40,41], square shoulder [42], and ramp potentials [43]. In Eq. (12) the ultrasoft potential of Ref. [1] was used. For clarity we report the potential form here:

$$
\begin{aligned}
\beta V(r) & =\frac{5}{18} f^{3 / 2}\left[-\ln \left(\frac{r}{\sigma}\right)+\frac{1}{1+\sqrt{f} / 2}\right], \quad r \leq \sigma \\
& =\frac{5}{18} f^{3 / 2} \frac{\sigma / r}{1+\sqrt{f} / 2} \exp \left[-\frac{\sqrt{f}(r-\sigma)}{2 \sigma}\right], \quad r \geq \sigma,
\end{aligned}
$$

where $\sigma$ is the interaction length, defined as the distance between two star centers, when the outermost blobs in the corona fully overlap. Theoretical structure factors were finally obtained by Fourier transformation of the pair correlation functions $g(r)$ :

$$
S(Q)=1+\frac{N}{V} \int d \mathbf{r} \exp (i \mathbf{Q} \cdot \mathbf{r})[g(r)-1] .
$$

\section{RESULTS AND DISCUSSION}

\section{A. Form factor and single particle properties}

In this section results concerning single particle properties, i.e., form factors $P(Q)$, will be presented. The micellar characterization was aiming at determining (i) the functionality $f$, as a function of DMF content of the solvent and (ii) the size of the core and corona of the micelles. Furthermore, the density distribution of the micellar corona was tested against Eq. (10) in order to verify the starlike morphology of the micelles.

Form factors $P(Q)$ have been measured in core and shell (corona) contrast conditions as a function of the DMF content of the solvent. For core contrast studies the polymer volume fraction in solution was in all cases $\phi \approx 0.25 \times 10^{-2}$. The desired scattering length density for core contrast condition was, in a first step, assumed to equal the scattering length density of PEO as calculated for Table I. Furthermore, since we could not exclude small uncertainties in chemical composition of $h / d$ material along the polymer chain and possible deviations of the density of PEO in solution as compared to bulk $[24,44,45]$, the core-contrast condition was determined in an experiment where the scattering length density of the solvent was changed starting from the fully deuterated solvent and adding step-by-step a droplet of hydrogenated solvent by using a Hamilton $10 \mu \mathrm{l}$ syringe, until no contribution from the corona could be detected. Figure 1 shows the SANS intensities measured at $8 \mathrm{~m}$ sample to detector distance. Intensities have been normalized to the polymer volume fraction $\phi$. Starting from the sample in pure $\mathrm{D}_{2} \mathrm{O}$, the forward scattering $[I(Q \rightarrow 0)]$ in the range $10^{-2} \AA^{-1}<Q<2 \times 10^{-2} \AA^{-1}$ shows an upturn which decreases with the addition of $\mathrm{H}_{2} \mathrm{O}$ until a plateau is reached after the third addition, corresponding to a $\mathrm{D}_{2} \mathrm{O}$ volume fraction in the solvent $\phi_{\mathrm{D}_{2} \mathrm{O}}=0.981$. Here the core contrast condition is achieved, providing a value of the scattering length density of the matching solvent $\rho_{c c}=6.31 \times 10^{10} \mathrm{~cm}^{-2}$. Further increasing the $\mathrm{H}_{2} \mathrm{O}$ amount produces a downturn of the

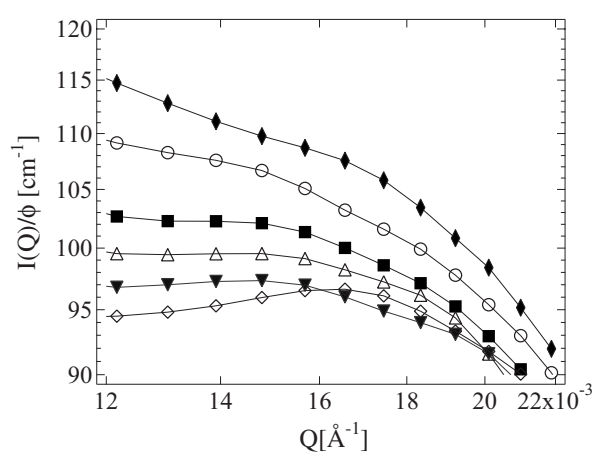

FIG. 1. SANS intensities from dilute PEP-PEO micellar solutions in $\mathrm{H}_{2} \mathrm{O} / \mathrm{D}_{2} \mathrm{O}$ mixtures. The intensities are normalized to polymer volume fraction $\phi$. The $\mathrm{D}_{2} \mathrm{O}$ volume fraction in the solvent $\left(\phi_{\mathrm{D}_{2} \mathrm{O}}\right)$ is $(\diamond)$ pure $\mathrm{D}_{2} \mathrm{O}, \phi_{\mathrm{D}_{2} \mathrm{O}}=0.999,(\bigcirc) \phi_{\mathrm{D}_{2} \mathrm{O}}=0.991$, ( $)$ $\phi_{\mathrm{D}_{2} \mathrm{O}}=0.985,(\triangle) \phi_{\mathrm{D}_{2} \mathrm{O}}=0.981,(\boldsymbol{\nabla}) \phi_{\mathrm{D}_{2} \mathrm{O}}=0.978$, and $(\diamond) \phi_{\mathrm{D}_{2} \mathrm{O}}$ $=0.974$.

forward scattering, meaning that the scattering length density of the solvent became smaller than that of PEO. In this case according to Eq. (6) the cross term in $|A(Q)|^{2}$ becomes negative changing the upturn of the forward scattering into a downturn. Additional details on this experiment can be found in [28].

The core radius $R_{c}$ and the functionality $f$ can be estimated from the position of the minimum in the form factor. Indeed, assuming a homogeneous compact spherical core, the form factor has its first minimum at

$$
Q R_{c} \approx 4.49 .
$$

Once obtained, $R_{c}$ from Eq. (15), $f$ derive from Eq. (9). Exemplary experimental core contrast form factors measured for two solvents with different DMF mole fraction, $x_{D M F}$ $=0.2$ and 0.5, are reported in Fig. 2(a). Qualitatively, by comparing the two form factors, it is evident that the position of the minimum is moving towards higher $Q$ values with increasing DMF content. According to Eq. (15), this means that $R_{c}$ is decreasing with increasing DMF content, and correspondingly the functionality $f$. The reduction of $f$ is confirmed by the reduced value of the forward scattering with increasing DMF content. Values of $R_{c}$ and $f$ obtained from the minimum position for all solvents are listed in Table II.

Figure 2(a) shows also fits to the compact core model described in Eqs. (5), (6), and (8). In the fit, the effect of experimental resolution was included, by convoluting the model function with the resolution function, as described in [46]. It should be noticed that since the resolution is different for the different instrumental setups used to cover the entire $Q$ range of data, the original fitted curves were composed of separated parts corresponding to each setup. In order to make the plots more clear, we decided to present them as a continuous curve instead of separated into parts. The only adjustable parameter in the fit was the core radius $R_{c}$. The functionality $f$ is obtained from $R_{c}$ according to Eq. (9). This also means that a good fit is obtained when both minimum position and forward scattering provide the same value of $f$. The small deviations between fitted curves and experimental 

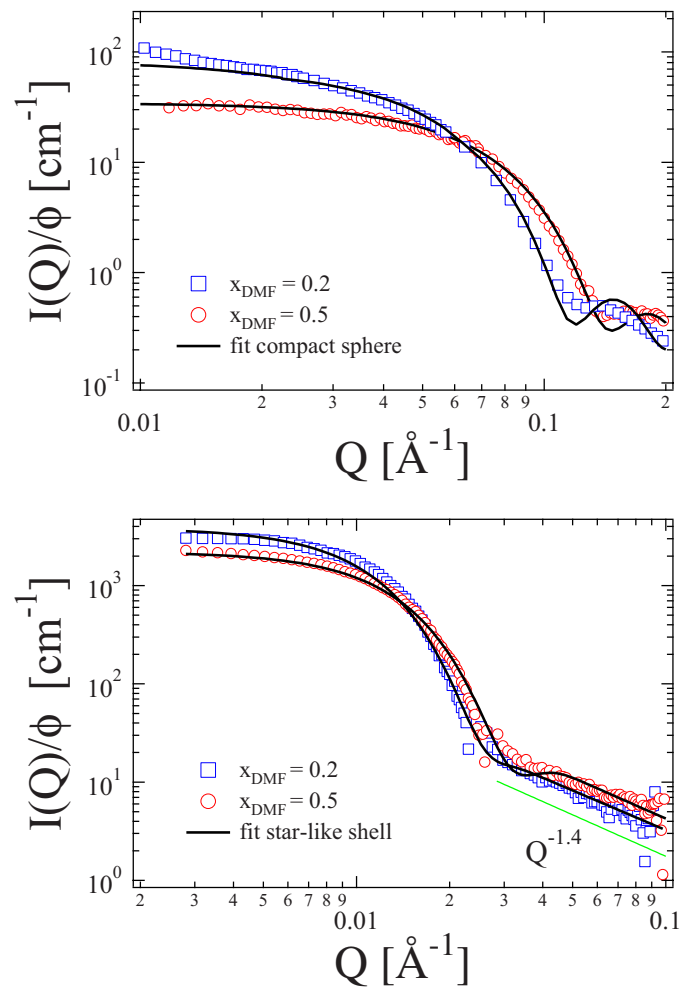

FIG. 2. (Color online) (a) Experimental core contrast form factors for $x_{D M F}=0.2$ and 0.5 solvent mixtures and fits to compact core model. (b) Experimental shell contrast form factors and fits to coreshell model; green line: $Q^{-1.4}$ scaling of $I(Q)$ in the high $Q$ range.

data observable around the minimum could arise from small polydispersity effects which would not seriously change the obtained values of $R_{c}$.

Additionally, experimental data for $x_{D M F}=0.2$ show some excess scattering in the region of $Q \leq 3 \times 10^{-2} \AA^{-1}$ that is not properly modeled. Such an excess scattering is most likely due to undesired instrumental effects, like an imperfect beam alignment. Such effects could be significant in the case of core-contrast form factors, since in such a contrast condition only a small part of the particle contributes to the coherent scattering (the small PEP core), therefore the overall scattering from the particles in a dilute solution is quite small compared to the overall scattering. Alternatively, the excess scattering could be attributed to residual shell contributions or to the presence of large scale structures, like clusters, in solution. We postpone the discussion of the last two effects to the section about interactions, since excess scattering was observed also in concentrated solutions. Values of $R_{c}$ and $f$ obtained from fits are also reported in Table II. The agreement with the set of results obtained from the minimum position is satisfactory.

Form factors in shell contrast condition are shown in Fig. 2(b) for the solvents $x_{D M F}=0.2$ and 0.5 . It can be noticed that, also in shell contrast, the forward scattering $[I(Q$ $\rightarrow 0)$ ] is decreasing with increasing DMF content, confirming the reduction of the micellar functionality. For $Q$ $\geq 0.03 \AA^{-1}$ the data show a clear power law dependence, which was found to be approximately $I(Q) \sim Q^{-1.4}$. For swollen polymer chains in good solvent condition a power law dependence $I(Q) \sim Q^{-1.7}$ is expected on the basis of the Flory exponent $\nu=0.588$ and the fact that at high values of $Q$ the scattering intensity from an object with fractal dimension $D$ scales as $I(Q) \sim Q^{-D}$, where $D=1 / \nu[47]$. The experimental value is smaller, in agreement with previous SANS experiments on PI and PB star polymers $\left[I(Q) \sim Q^{-1.5}\right][48-50]$, where the larger value of $\nu$ was attributed to a stretched configuration of the chains in star polymers due to geometric-steric constraints. Data were modeled according to the core-shell model of Eqs. (5) and (6), assuming a starlike density distribution of the micellar corona according to Eq. (10). In order to stabilize the values of $R_{m}$ in the fits, the smearing parameter of the Fermi function, $\sigma_{m}$, was kept fixed at $10 \%$ of the micellar radius, according to previous investigations of asymmetric PEP-PEO micelles [16]. Since for us the parameter of interest in the modeling was the micellar radius $R_{m}$, we did not go in depth using a microscopic description of the blob scattering, therefore we used the Beaucage function described in Eq. (11) to model the scattering intensity at high $Q$. Blob intensity prefactors were found to be of order $10^{-23} \mathrm{~cm}^{3}$, the blob size was varying in the range $\xi=60-80 \AA$, while the fractal exponent was found to be approximately $D=1.4$ in all cases. Values of $R_{m}$ obtained from fits are reported in Table II. The obtained values show a contraction of the micellar corona with increasing DMF content, consistent with the decrease of the second virial coefficient $A_{2}$ with increasing DMF content observed in Ref. [17]. The decreasing functionality with increasing DMF content could only contribute slightly to the contraction of the micellar corona. Indeed let us define the reduced surface covering of the micellar core according to [34], $\sigma_{\text {red }}=\left(\pi f R_{g 0}^{2}\right) / 4 \pi\left(R_{c}+R_{g 0}\right)^{2}$, where $R_{g 0}$ is the radius of gyration of a chain in the micellar corona. If one calculates $\sigma_{\text {red }}$ using the values of $R_{c}$ from Table II and of $R_{g 0}$ from Ref. [17], one obtains a decrease from $\sigma_{\text {red }}=14$ at $x_{D M F}=0$ to $\sigma_{\text {red }}=8$ at $x_{D M F}=0.5$, which means that the corona is still in the brush regime $\left(\sigma_{\text {red }} \gg 1\right)$.

The good quality of the fits, based on the starlike profile of Eq. (10), confirms the starlike architecture of the micellar corona.

The starlike morphology of the micelles was also directly verified by a comparison to the form factor of PB star polymers with nominal functionality $f=64$, as shown in Fig. 3 . The $Q$ vectors of the two data sets were rescaled to the respective radii of gyration, $R_{g}=135 \AA$ for the micelles according to Table II and $R_{g}=44.3 \AA$ for the PB stars [3]. The SANS intensities were rescaled to the respective volume fractions, contrast $\left(\Delta \rho^{2}\right)$, and molecular volumes $\left(V_{w}\right)$ of polymer chains. The two curves accurately overlap in the whole $Q$-range, thus confirming the starlike morphology of PEP-PEO micelles.

The starlike features of the micellar corona can be emphasized by using a generalized Kratky representation of the data, i.e., by plotting $I(Q) Q^{1 / \nu}$ vs $Q$. For branched polymers like star polymers, this representation shows a characteristic peak whose height reduces with decreasing functionality. This behavior was experimentally observed for star polymers [48]. Figure 4 shows shell contrast form factors corresponding to different values of $x_{D M F}$ in generalized Kratky repre- 


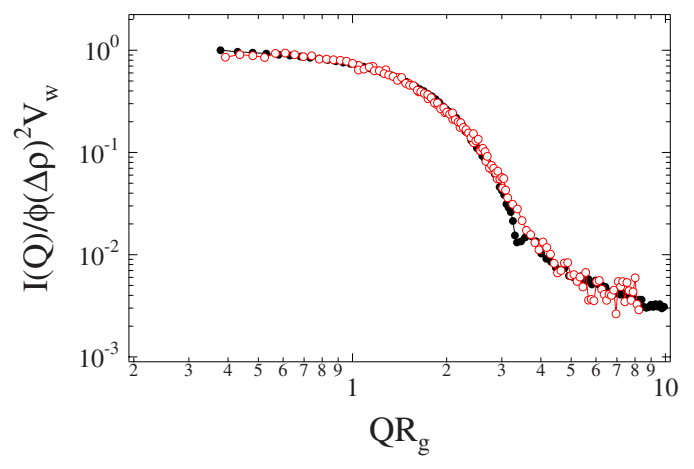

FIG. 3. (Color online) Comparison between shell contrast form factor of PEP-PEO micelles with $f=63$ (closed symbol) and form factor of $\mathrm{PB}$ stars with nominal functionality $f=64$ (open symbol).

sentation, where $1 / \nu$ has chosen to be equal to 1.4. A clear characteristic peak is present, whose height is strongly decreasing from pure water to $x_{D M F}=0.5$, corresponding to the reduction of micellar functionality. Data are merging into a plateau in the region of higher $Q$, which corresponds to the regime where $Q^{-1.4}$ scaling of intensities was observed. The inset of Fig. 4 presents, in Kratky representation, the comparison between the form factor of PEP-PEO micelles with $f=63$ and the form factor of PB stars with nominal $f=64$.

The Kratky representation provides an additional independent way to estimate the radius of gyration of the micelles. In the region around the peak, the form factor can be approximated by the Gaussian star form factor of Benoit [51]:

$$
P(Q)=\frac{2}{f v^{4}}\left(v^{2}-\left[1-\exp \left(-v^{2}\right)\right]+\frac{f-1}{2}\left[1-\exp \left(-v^{2}\right)\right]^{2}\right),
$$

where

$$
v=\sqrt{f /(3 f-2)} Q R_{g} .
$$

This form factor is in principle describing star polymers un$\operatorname{der} \Theta$ solvent conditions, but excluded volume effects for swollen chains are not affecting the $Q$ range around the peak.

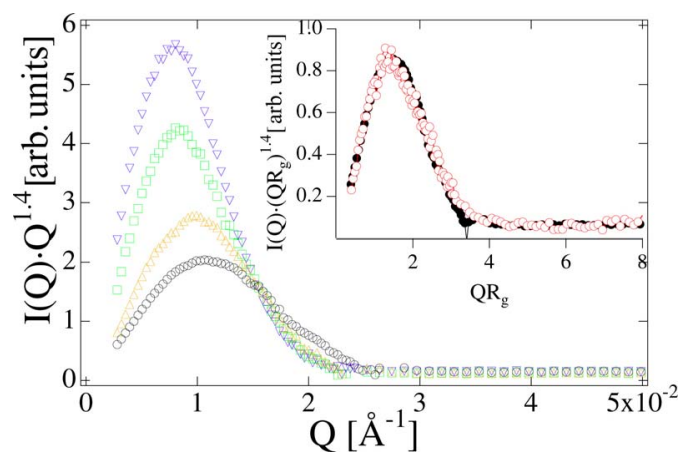

FIG. 4. (Color online) Generalized Kratky representation of shell contrast form factors for different values of DMF content: $(\nabla)$ $x_{D M F}=0.0,(\square) x_{D M F}=0.1,(\triangle) x_{D M F}=0.2$, and $(\bigcirc) x_{D M F}=0.5$. Inset: Kratky representation of form factors of PEP-PEO micelles with $f=63$ (closed symbol) and PB stars with $f=64$ (open symbol).
TABLE III. Left: Comparison between values for the radius of gyration obtained from structure factor modeling of solutions below $\phi^{*}\left(R_{\sigma}\right)$, from form factor analysis $\left(R_{g}\right)$, and from the Benoit model applied to Kratky representation of shell-contrast form factors $\left(R_{g}^{k r}\right)$. Right: $a_{b c c}$ and $a_{s c}$ values obtained from crystalline samples with $f=63\left(x_{D M F}=0.5\right)$ and different values of the packing fraction $\eta$ $=(\pi / 6) N_{z} \sigma^{3}$, where $N_{z}$ is the particle number density and $\sigma$ the interaction length. Values of $2 R_{m}$ are reported for comparison. Analogous results, not shown here, were obtained for samples with

\begin{tabular}{|c|c|c|c|c|c|c|c|c|}
\hline$f$ & $\begin{array}{l}R_{\sigma} \\
(\AA)\end{array}$ & $\begin{array}{c}R_{g} \\
(\AA)\end{array}$ & $\begin{array}{l}R_{g}^{k r} \\
(\AA)\end{array}$ & $\eta$ & $\begin{array}{l}a_{b c c} \\
(\AA)\end{array}$ & $\begin{array}{l}a_{s c} \\
(\AA)\end{array}$ & $\begin{array}{c}2 R_{m} \\
(\AA)\end{array}$ & $f_{b c c}$ \\
\hline 63 & 127 & 140 & 144 & 0.21 & 381 & 270 & 377 & 82 \\
\hline 67 & 136.5 & 145 & 148 & 0.23 & 367 & 260 & 375 & 82 \\
\hline 73 & 140 & 155 & 155 & 0.24 & 367 & 260 & 372 & 90 \\
\hline 82 & 154 & 169 & 166 & 0.26 & 341 & 241 & 368 & 81 \\
\hline 94 & 161 & 184 & 198 & 0.28 & 341 & 241 & 362 & 91 \\
\hline \multirow[t]{2}{*}{136} & 192 & 200 & 202 & 0.31 & 330 & 233 & 356 & 94 \\
\hline & & & & 0.41 & 309 & 218 & 336 & 112 \\
\hline
\end{tabular}
$f=67\left(x_{D M F}=0.4\right) . f_{b c c}$ values are calculated according to Eq. (20).

The position of the maximum of the Benoit form factor, $v_{\max }$, can be calculated from the first derivative of expression (16) with respect to $v$, for each value of the functionality $f$, fixed to the experimental values of Table II. Values of $v_{\max }$ in the range $v_{\max }=0.94-1.01$ were found depending on $f$. Using these values and positions of the maximum from experimental data of Fig. 4, the micellar radius of gyration $R_{g}$ was estimated for each functionality. Values of $R_{g}$ obtained from the Benoit model applied to form factors in Kratky representation are listed in Table III. Values of $R_{g}$ from the Benoit model are in good agreement with values independently obtained from the core-shell model and interaction length $\sigma$ (next section).

On the basis of the results of characterization of PEP-PEO micelles in dilute solution and comparison to regular star polymers, we can conclude that for what concerns internal structure and morphology, our micellar system can be described as a starlike system where the functionality can be easily adjusted through changes in solvent composition.

\section{B. Structure factor and interactions}

Interactions between micelles were investigated through interparticle structure factors $S(Q)$ obtained by SANS. Experimental structure factors for the six different functionalities were extracted from SANS intensities measured in core contrast, as explained in Sec. II B 1. Structure factors for several values of polymer volume fraction have been measured for each functionality. A direct connection between interactions in PEP-PEO micelles and in regular star polymers was obtained by comparing structure factors obtained for micelles with $f=63$ (water/DMF mixture with molar fraction $\left.x_{D M F}=0.5\right)$ and a corresponding PB star polymer in methylcyclohexane with (nominal) 64 arms [3]. Structure factors measured at different values of the ratio $\phi / \phi^{*}$ have been compared, with $\phi^{*}=3 /\left(4 \pi R_{m}^{3}\right) \times\left(f M_{w} / \bar{\delta} N_{A}\right)$ being the over- 

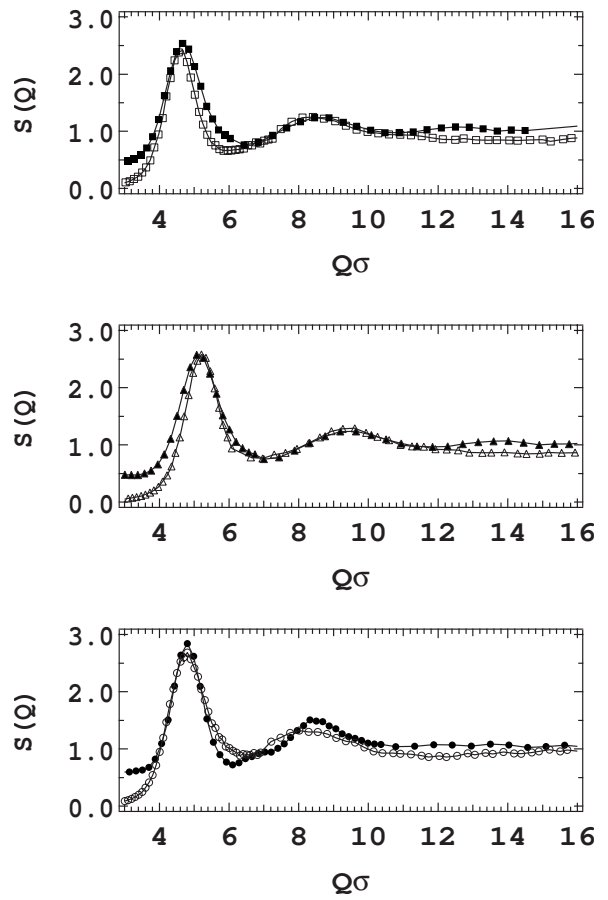

FIG. 5. $S(Q)$ for PEP-PEO micelles with $f=63$ (closed symbols) compared to a 64-arm (nominal) PB star (open symbols), at three different values of the ratio $\phi / \phi^{*}$ : (a) $\phi / \phi^{*} \approx 0.82$, (b) $\phi / \phi^{*} \approx 1$, and (c) $\phi / \phi^{*} \approx 1.30$. Solid lines are a guide for the eyes.

lap volume fraction and $\bar{\delta}$ the average density. Figure 5 shows the comparison between structure factors of PEP-PEO micelles and PB star polymers for three values of $\phi$, below, at and above the overlap volume fraction: The main features of the star $S(Q)$ with respect to peak positions and heights are well-reproduced below and at the overlap volume fraction, as depicted in plots (a) and (b) of Fig. 5. The fact that a third peak in the region of large $Q \sigma$ is observed for the micellar system but not for the PB star is due to the fact that for the PB star this peak is masked by the diffuse blob scattering of the polymer arms [31].

Above $\phi^{*}$, while the first peaks still overlap, differences evolve between the star and the PEP-PEO micelles for larger values of $Q \sigma$ [Fig. 5(c)]. In particular the $S(Q)$ of the micelles presents an additional peak where the structure factor of the stars has the first minimum $(6<Q \sigma<7)$. As it will be explained in more detail later, the observed additional peak in the micellar $S(Q)$ is related to the formation of a crystalline phase in the micellar system above $\phi^{*}$, not observed for the PB stars.

The larger width of the micellar $S(Q)$ around the first peak can be attributed to the different resolution of the D11 spectrometer $(6 \AA$ wavelength, $10 \%$ wavelength spread, 1 $\times 1 \mathrm{~cm}^{2}$ detector pixel size, $30 \times 30 \mathrm{~mm}^{2}$ squared collimation aperture, and $13 \mathrm{~mm}$ diameter of sample aperture), where micellar data have been measured, and of the PAXY spectrometer (10 ̊ wavelength, $13 \%$ wavelength spread, $0.5 \times 0.5 \mathrm{~cm}^{2}$ detector pixel size, $13 \mathrm{~mm}$ diameter of collimation aperture, and $7.6 \mathrm{~mm}$ diameter of sample aperture), where PB star data have been measured. In all three comparisons, in the low $Q$ region the micellar $S(Q)$ shows some increase indicating some excess scattering. Such excess scattering might arise either from (i) (inherent) mesoscopic heterogeneities as also found in experiments with purely repulsive star polymer solutions [52,53] or (ii) from weak attractive interactions due to residual dispersion forces, i.e., decreasing solvent quality with increasing $x_{D M F}[17]$ or (iii) residual contributions from the shell form factor.

In the first case, this result only further supports the analogy between PEP-PEO micelles and star polymers. It should be noticed that the analogy refers to good solvent conditions but is not completely athermal, in agreement with the cited Refs. [52,53]. Indeed, as one can see in Fig. 5, no signature of such heterogeneities appear in PB star polymers in methylcyclohexane, which is an athermal solvent for PB. In the second case, it has been shown that the presence of an attractive interaction (for example, of van der Waals type) in addition to the repulsive potential of Eq. (13) does not substantially influence the freezing and the glass transition of the system, if the width of the attraction well is sufficiently large $[39,54]$, while a shift of the MCT ideal glass transition line towards larger packing fractions is expected for a shorter range of attraction [54]. Our results on the phase diagram, presented later in this section, show that dynamical arrest in the system happens at volume fractions comparable or smaller than expected for purely repulsive star polymers, thus pointing in the direction of absence of attractive interactions, or presence of rather long-ranged attractive interactions in our system. Finally the presence of residual contributions from the shell in the low- $Q$ range might arise from different mechanisms of water-heavy water adsorption around the soluble partially deuterated PEO block [55-57]. The origins of such mechanisms are still under debate and a quantitative discussion of these effects on the basis of our data is under investigation. It has to be noted though that spurious contributions from the shell would not affect the range of $Q$ where the structural features, i.e., peaks of the structure factor $S(Q)$ appear, as also confirmed by the core contrast experiment of Sec. III A.

Excess scattering affects the low $Q$ regime of all data from concentrated solutions, giving rise to a discrepancy between experimental data and theory calculations in this regime. Here we will focus on the $Q$ regime where the structural features corresponding to pair interactions between single particles are present. As will be shown below on the basis of our analysis, whatever the origin of such excess scattering is, the low- $Q$ scattering does not have major relevance on the quantitative interpretation of $S(Q)$ for $Q$ $\geq 10^{-2} \AA^{-1}$.

Interactions in PEP-PEO micellar solutions were also compared to theoretical predictions for regular star polymers. We will describe in detail the modeling procedure that was used to model SANS data from the investigated samples. It should be noticed here that, since we have shown that interactions in our system reproduce those of real star polymers, a description of the interactions on the basis of a hard-sphere potential was excluded from the beginning.

The modeling was obtained in a two-step procedure. In the first step, theoretical $S(Q)$ were calculated, according to the procedure described in Sec. II B 2, for a fixed $f$ and different values of the packing fraction $\eta$, that are calculated 
a)

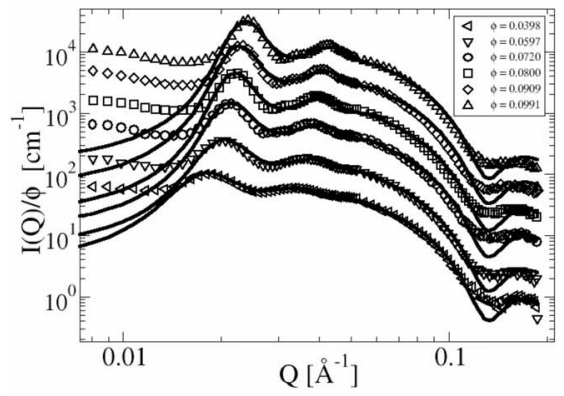

c)

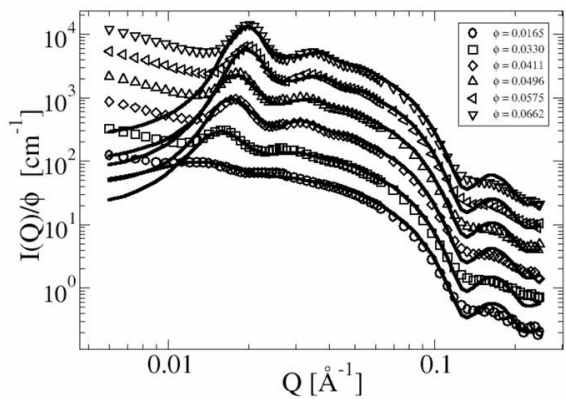

b)

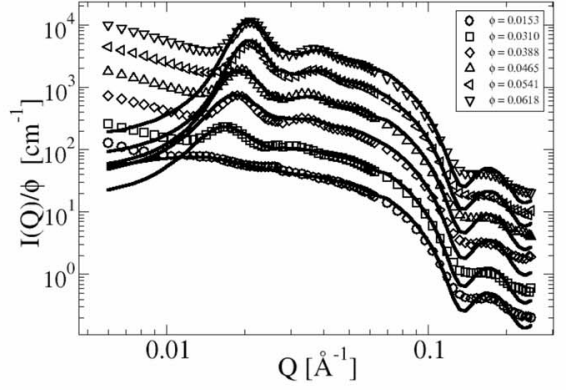

d)

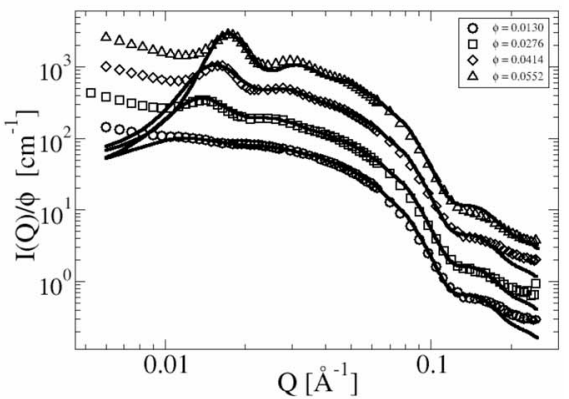

FIG. 6. Comparison between experimental SANS intensities (points) and theory (lines) for four different functionalities: (a) $f=67$, (b) $f=73$, (c) $f=82$, and (d) $f=94$, and different volume fractions $\phi$ (in legends). For clarity, in each plot intensities have been shifted with respect to the data with the smallest volume fraction.

according to $\eta=(\pi / 6) N_{z} \sigma^{3}$, where $N_{z}$ is the particle number density. The obtained structure factors were compared to experimental ones at fixed volume fraction by rescaling the theoretical $Q \sigma$ abscissa to the experimental $Q$-range. This means that for each different $\eta$ we obtained a different value of $\sigma$. It has to be noticed that the experimental packing fraction in terms of $\sigma$ cannot be calculated a priori on the basis of the measurements, since $\sigma$ can only be obtained by modeling the experimental structure factor. The second step consisted of determining the best description of the experimental structure factor among the selected theoretical ones. To do that, it was necessary to take into account the effect of experimental resolution, which is affecting the peak height and width of the experimental $S(Q)$.

Since the experimental $S(Q)$ cannot be easily deconvoluted from resolution effects, it is then preferable to convolute the theoretical model for the overall SANS coherent cross section $I(Q) \sim P(Q) S(Q)$ to the resolution function, as mentioned for the form factor analysis. For $P(Q)$ we assumed the compact core model, fixing the parameters to $f$ and $R_{c}$ values obtained from the characterization of the micelles. The validity of this assumption was explained in Sec. II B 1. For each experimental $I(Q)$, the selected different theoretical structure factors, corresponding to different values of $\sigma$, were tested against experimental data. The result of comparison between theory curves where resolution effects are taken into account and the measured cross section allows us to select the theoretical structure factor which gives the best description of the experimental data. The best description of the data is selected as the one where the height and width of the structural peaks is more accurately reproduced. From the chosen theoretical structure factor the value of $\sigma$ for a given experimental packing fraction is obtained.

Use of potential of Eq. (13) to calculate $S(Q)$ was proven to give satisfactory description of experimental data for the full range of functionalities and volume fractions measured. Examples of comparisons between theory and experiments are shown in Fig. 6. In the same figure, in each single plot related to a fixed functionality $f$, it can be again observed that at all different volume fractions the position of the minimum of the form factor, visible in the range $Q \geq 0.1 \AA^{-1}$, does not change with increasing volume fraction, confirming the stability of the micellar morphology at all volume fractions.

The $\sigma$ parameter is defined in the theoretical model as the distance between the two star centers, when the outermost blobs in the corona fully overlap. The radius of this blob, from geometry, is simply $R_{b} \approx \sigma / \sqrt{f}$. Since below $\phi^{*}$ the particles do not overlap, a star radius can be defined as the half of $\sigma$ plus the radius of the outermost blob:

$$
R_{\sigma}=\frac{\sigma}{2}+\frac{\sigma}{\sqrt{f}} .
$$

Using Eq. (18), $R_{\sigma}$ was calculated for all measured samples, $f$ was taken from the form factor analysis.

According to scaling theory, the size of a star should not be affected by concentration effects until the star coronas start to overlap [58]. This means that below the overlap volume fraction $\phi^{*}, R_{\sigma}$ should stay constant at fixed $f$. Values of $R_{\sigma}$ obtained for samples below $\phi^{*}$ are plotted as a function of $\phi$ in Fig. 7, for all measured functionalities. As it can be 

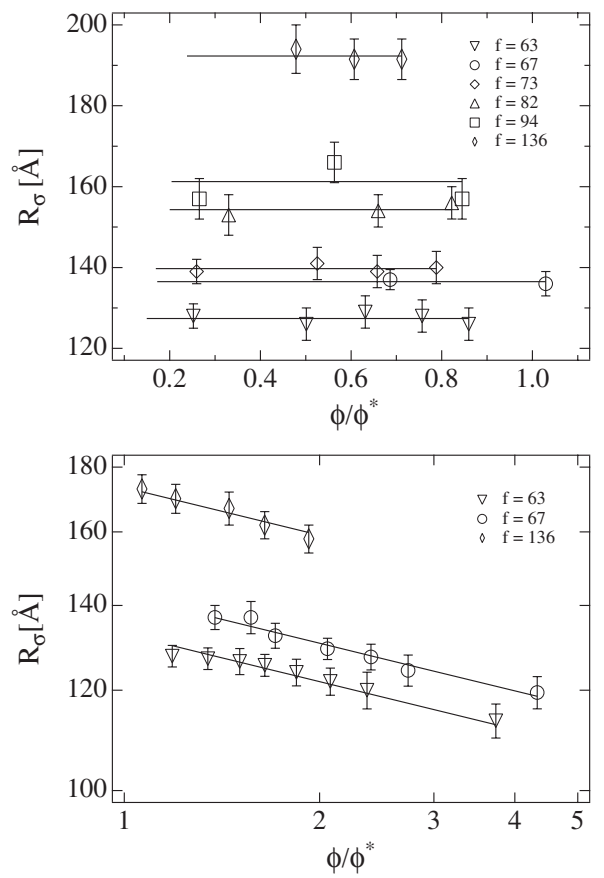

FIG. 7. Scaling behavior of $R_{\sigma}$ with $\phi$ for samples with polymer volume fraction below $\phi^{*}$ (upper plot) and above $\phi^{*}$ (lower plot). For each $f R_{\sigma}$ is constant below $\phi^{*}$ and scales as $\phi^{-1 / 8}$ above $\phi^{*}$.

noticed, in all cases data related to a single $f$ value are distributed with small deviations around an average value $R_{\sigma}$, in agreement with scaling theory predictions. Moreover, data can be compared to the radius of gyration obtained in the very dilute regime, i.e., from the form factor analysis. From that analysis the micellar radius $R_{m}$ was obtained as the cutoff distance of the Fermi function for the starlike density profile in the corona, see Eq. (10). $R_{g}$ can be calculated from $R_{m}$ according to

$$
R_{g}^{2}=\frac{\int_{0}^{R_{m}} n(r) r^{4} d r}{\int_{0}^{R_{m}} n(r) r^{2} d r},
$$

where $n(r)$ corresponds to the expression in Eq. (10). Values of $R_{g}$ are in good agreement with $R_{\sigma}$, as it can be noticed from Table III.

When the particles start to interpenetrate, the star coronas are shrinking. The Daoud-Cotton model predicts that the radius of a star polymer scales as $\sim \phi^{-1 / 8}$ with increasing polymer volume fraction above $\phi^{*}[9,58]$. This scaling behavior was tested only for samples with $f=63,67$, and 136 , where a sufficient number of samples above $\phi^{*}$ were measured. For all three cases the agreement between experimental data and scaling theory predictions is excellent, as shown in Fig. 7. It should be noticed that, while the range of volume fractions investigated is not large enough to fully establish scaling predictions, it is much larger than in previous studies of regular star polymers [48]. We can conclude that the interaction length $\sigma$ obtained by describing experimental $S(Q)$ via

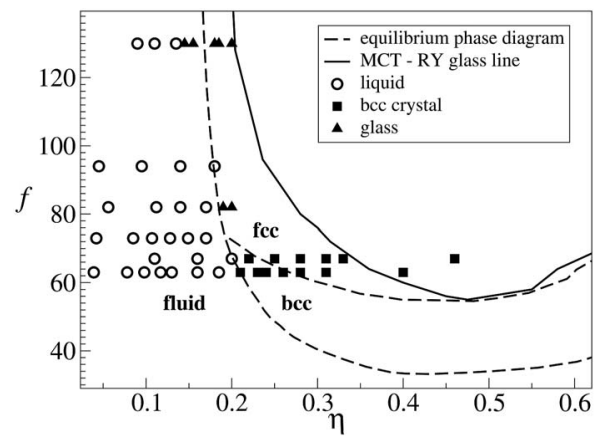

FIG. 8. Experimental phase diagram (symbols) of starlike micelles vs theoretical phase diagram (lines) of star polymers. $(\bigcirc)$ liquid, ( $\boldsymbol{\square})$ bcc crystal, and $(\boldsymbol{\Delta})$ gels. The dashed line represents the equilibrium phase diagram from Ref. [5]. The solid line is the RYideal MCT glass line from Ref. [6].

the ultrasoft potential proposed for regular star polymers are within error bars quantitatively given by the molecular size of the single starlike PEP-PEO micelle.

\section{Phase diagram}

The values of $\sigma$ obtained from comparison between theory and experiments have been used to recalculate the packing fraction $\eta$ from the given experimental particle number density $N_{z}$. The evaluation of $\eta$ is essential in order to compare the experimental and theoretical phase diagram. For $\phi<\phi^{*}$ the average value of the interaction length displayed in Fig. 7 was used, while for $\phi>\phi^{*}$ we took the individual values of $\sigma$ obtained for each separate volume fraction, since $\sigma$ varies with $\phi$.

Experimental points with coordinates $(\eta, f)$ were then located in the theoretical phase diagram of Ref. [5]. The resulting diagram is presented in Fig. 8. Good agreement between theory and experiments was obtained for the liquid-solid boundary in the whole range of functionalities and packing fractions. In particular the liquid-to-bcc crystal transition for $f<70$ is perfectly reproduced experimentally. The packing fraction for crystallization was found to be $\eta_{c} \approx 0.21$.

Experimental evidence for the formation of crystalline states comes from the observation of Bragg peaks in the two-dimensional (2D) SANS pattern of the detector, as shown in Fig 9. Moreover, structure factors of samples presenting Bragg peaks have a first peak which equals or exceeds the minimum value for freezing transition, 2.8, predicted by the Hansen-Verlet criterion [59]. It should be noted that the effect of experimental resolution is that of reducing the peak height, therefore the Hansen-Verlet criterion is certainly fulfilled.

The crystalline lattice can be determined on the basis of the relative position of the first three peaks of the structure factor. It can be noticed that, corresponding to the onset of crystallization, an additional peak at intermediate distances appears in the structure factors, as shown in Fig. 9. The peak ratio with respect to the first peak is $1: \sqrt{2}: \sqrt{3}$, characteristic of a bcc or simple-cubic (sc) lattice [60]. The A15 lattice [61] can be excluded, since its additional reflection at $\sqrt{5 / 2}$ was 


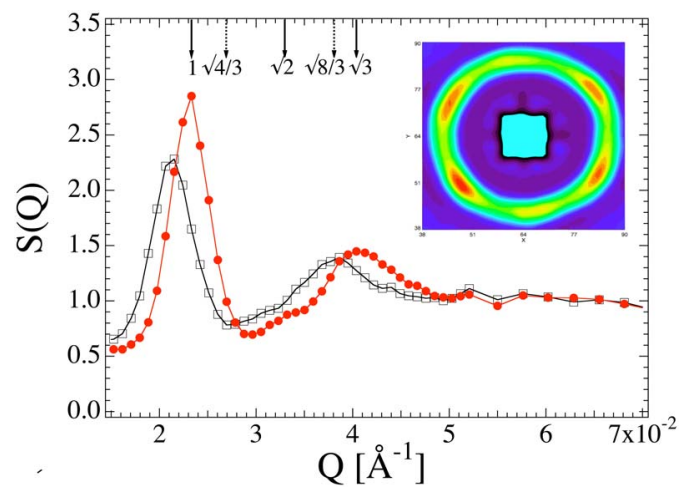

FIG. 9. (Color online) Liquid (open symbol, $\phi=0.072$ ) to crystal (closed symbol, $\phi=0.091$ ) transition observed for samples with $f=67$ : A structure factor above $\phi^{*}$ presents a first peak larger than 2.8 and an additional second peak, the ratio between the three peaks is $1: \sqrt{2}: \sqrt{3}$. Inset: Bragg peaks observed in a 2D SANS pattern of the detector, for $f=63$ and $\phi=0.16$.

not observed. It is expected to occur in a region where the form factor is close to unity. In order to select between the bcc and the sc lattice, we calculated the lattice parameter $a$ for the two options, $a_{b c c}=2 \pi \sqrt{2} / Q_{\max }$ and $a_{s c}=2 \pi / Q_{\max }$. At the overlap volume fraction the lattice parameter should be equal to the double of the micellar radius $R_{m}$ and decrease for larger volume fractions because particles start to interpenetrate. This is the trend of $a_{b c c}$ with increasing $\phi$ above $\phi^{*}$ whereas the values of $a_{s c}$ are always smaller than $2 R_{m}$, see Table III. Therefore the cubic lattice was identified as bcc.

Additionally it may be speculated that the ability of the system to crystallize for functionalities $f<70$ is connected to the significant copolymer exchange between micelles recently measured in time-resolved SANS measurements $[25,26]$. Probably, this facilitates the packing of micelles in ordered structures with increasing volume fraction, as observed also for other micellar systems $[62,63]$. It should be noticed though that still the critical packing fraction for crystallization should be determined by the interaction potential between micelles at a specific functionality, when the functionality does not change with polymer volume fraction: In our case indeed the average micellar functionality is unchanged over the whole range of polymer volume fractions, as already shown from the stability of the form factor minimum (Fig. 6), and we were able therefore to relate the observed liquid-to-bcc crystal transition directly to the interaction potential predicted for star polymers.

Still considering the range of functionalities $f<70$, further increasing the polymer volume fraction in the solid phase we could not observe any evidence of transition to an fcc lattice. At the moment we are missing a clear explanation of this phenomenon. Theory predicts in this functionality range a rather small free energy difference between bcc and fcc lattices: for example, for $f=64$ and $\eta=0.5$ the lattice energy sum for bcc is $E / k_{B} T=94.65$ and for fcc is $E / k_{B} T$ $=94.07$ [64]. Such an energy difference is enough to stabilize fcc against bcc, however, a metastable bcc phase could be formed as a precursor of the equilibrium fcc phase, according to the Ostwald's step rule [65]. Such a metastable bcc phase was observed for PS-PI micelles [62] and it was shown to have a lifetime of the order of hours.

In our experiments, solutions corresponding to packing fractions, where fcc lattices would be expected as equilibrium states, were measured after a time period ranging from 1 to 2 weeks from sample preparation. Since only bcc phases were observed, this would mean that the lifetime of such a metastable state would be in our case at least longer than 2 weeks. A different explanation would be that of formation of mixed phases, consisting of equilibrium bcc crystalline regions containing a fraction of the total number of micelles and dynamically arrested regions containing the remaining particles. Far enough from the glass transition one would expect an almost homogeneous bcc crystalline phase, while increasing the packing fraction towards the glass transition more arrested regions should be formed. Formation of such a mixed phase would prevent the establishment of the underlying equilibrium fcc phase. Such a speculation could be tested by checking the homogeneity of the crystalline bcc phases at packing fractions where an fcc phase would be expected. This could be done on the basis of simple geometrical considerations: In the case of a homogeneous bcc phase, the sample volume is given by the number of cubic cells times the volume of a cell $\left(a_{b c c}^{3}\right)$. Since in a bcc crystal there are two particles per unit cell, the number of cells is simply half of the number of particles. For a monodisperse starlike micelle, each particle is constituted of $f$ polymer chains, which means that the number of particles is equal to the number of polymers divided by $f$. Therefore we could recalculate the micellar functionality according to the relation

$$
f=\frac{N_{A} \delta_{P E P-P E O} \phi a_{b c c}^{3}}{2 M_{w}}
$$

where $\delta_{P E P-P E O}$ is the average polymer density. Values of functionality calculated according to Eq. (20) for samples with $f=63$ and polymer volume fractions where fcc phases are expected are listed in Table III. The values are in all cases significantly larger than those established on the basis of the form factor analysis. This suggests that the observed bcc crystalline phases are not homogeneous. Moreover, the difference between the functionalities obtained from Eq. (20) and those obtained from the form factor analysis is almost constant in the region far away from the glass transition, where bcc crystals are expected from theory, while it starts to increase in the region, approaching the glass transition, where fcc crystals are expected.

For $f>70$, when crossing the overlap volume fraction $\phi^{*}$, no crystals are formed. Indeed no Bragg peaks were observed in SANS measurements, and the obtained structure factors present first peaks that do not fulfill the HansenVerlet criterion, as shown in Fig. 10. Nevertheless for $\phi$ $\geq \phi^{*}$ all samples were found to be in a solid state. Evidence for formation of solid, noncrystalline states for $f>70$ was obtained from tube inversion. By tube inversion we checked the tendency of the sample to flow: For all solid samples we could not observe any flow over a time scale of the order of 


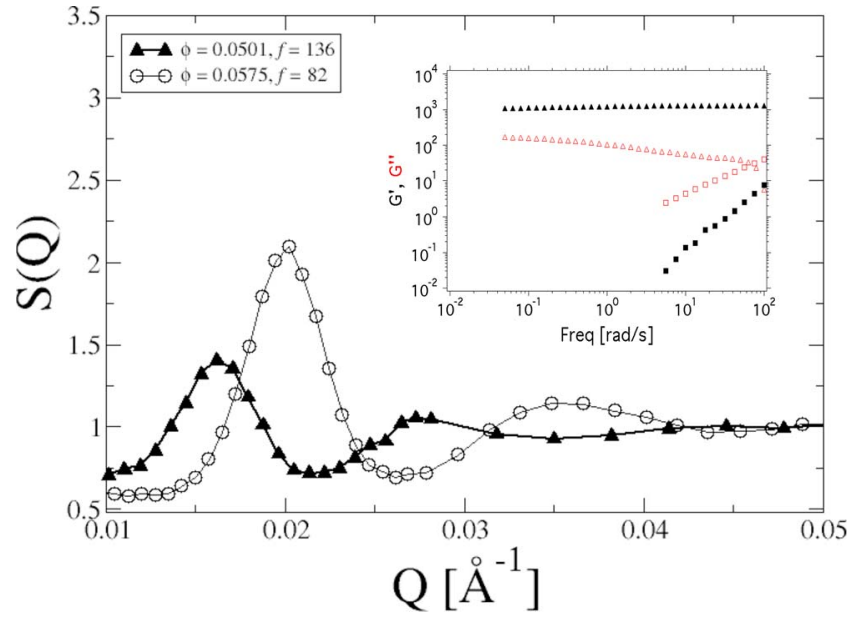

FIG. 10. (Color online) Structure factors for samples in the glassy solid state for $f=82(\bigcirc)$ and $f=136(\boldsymbol{\Delta})$ at $\phi \geq \phi^{*}$. Inset: Dynamic frequency sweeps for a liquid $(\phi=0.03, \mathbf{\square})$ and a solid $(\phi=0.035, \boldsymbol{\Delta})$ sample of hydrogenated PEP-PEO micelles in water.

a week. The temperature of the sample was kept constant by means of a liquid bath. Results of tube inversion experiments are supported by oscillatory rheology measurements performed for solutions of the analogous (same block ratio) fully hydrogenated PEP-PEO block copolymer. The inset of Fig. 10 shows results of dynamic frequency sweeps for two solutions of the hydrogenated PEP-PEO block copolymer in pure water $(f=120)$, one slightly below the overlap volume fraction and one slightly above it. Below $\phi^{*}$ the response is characteristic of a viscous fluid, with the loss modulus $G^{\prime \prime}$ larger than the storage modulus $G^{\prime}$ and a determined frequency dependence. Above $\phi^{*}$ one instead observes a typical solidlike response, with the storage modulus larger than the loss modulus.

Formation of disordered arrested states above the overlap volume fraction $\phi^{*}$ for $f \geq 70$ has been also reported for PB stars $[10,53]$. The dynamical arrest of the system was attributed to formation of clusters when the arms in the outermost blobs of the particles start to overlap. Indeed in case of a sufficiently large segment density in the corona, the geometric confinement can strongly suppress the chain dynamics, leading to formation of geometric constraints and consequently of clusters. Even a small number of clusters could induce a macroscopically arrested state [53].

Therefore formation of clusters approaching the overlap volume fraction $\phi^{*}$ could lead to dynamical arrest of the system at smaller values of the packing fraction $\eta$ than predicted by MCT [6], where the cage effect suppresses the dynamics when the particles already overlap significantly $\left(\phi>\phi^{*}\right)$.

Moreover, clusters can induce in the samples an "effective" polydispersity in the sample [66] that suppresses the underlying fcc phase. In addition, the absence of significant block copolymer exchange between micelles hampers the ordering into the fcc phase. For $f<70$ on the contrary the glass transition is predicted for packing fractions well above the crystallization boundary. Therefore this effect does not play an important role and we could observe crystalline phases.

\section{CONCLUSIONS}

We have presented a detailed investigation of single particle properties and interactions of starlike PEP-PEO copolymer micelles. The micellar system was first characterized by SANS combined with contrast variation, in terms of single particle properties, determining the functionality and the core and shell radii as a function of DMF content of the solvent. It was shown that the shell contrast form factor of the micellar corona can be modeled assuming a starlike density profile. The starlike morphology of the micelles was also directly confirmed by comparison to form factor data of a corresponding (same functionality) PB star polymer and evidenced in a Kratky representation. On the basis of single particle properties we could therefore conclude that the micelles are an easily tunable system having a starlike architecture.

The interactions in the system were found to reproduce those observed for PB star polymers, as obtained by a direct comparison of the experimental structure factors of the two systems below, at and above the overlap concentration. Moreover, it was shown that the pair potential describing the interactions between star polymers correctly describes also the interactions between PEP-PEO micelles. Therefore we could conclude that PEP-PEO micelles are a model system for both single particle properties and interactions of real star polymers. Hence we used PEP-PEO micelles to investigate the phase diagram of real star polymers. The agreement between experimental and theoretical phase diagram was found to be generally satisfactory, in particular the liquid-solid boundary is correctly reproduced in experiments for all functionalities. For $f<70$ the solid phase was identified as a bcc crystalline phase. The critical packing fraction for a liquidto-bcc crystal transition for $f<70$ shows excellent agreement with theory. We suggest that the observed bcc phases are not homogeneous, rather coexisting with dynamically arrested regions which grow in size approaching the MCT glass transition line. For $f>70$ the solid phase was identified as a dynamically arrested state. We suggest that the arrested state arises from dynamical frustration of the chain motions due to the confinement of the polymer chains of the corona when the particles start to overlap. This is in agreement with regular star polymers with sufficiently dense corona. This effect was not taken into account in MCT calculations, but explains the early onset of gelation observed in experiments compared to theory. Moreover, we speculate that an effective polydispersity induced by cluster formation could prevent the occurrence of the equilibrium fcc crystalline phase.

\section{ACKNOWLEDGMENTS}

We acknowledge the allocation of SANS beam time by Forschungszentrum Juelich (FZJ), Institute LaueLangevin (ILL), and Laboratoire Leon Brillouin (LLB) and assistance during the SANS experiments by A. Radulescu 
(FZJ), P. Lindner (ILL), and L. Noirez (LLB). We acknowledge helpful discussions with C.N. Likos and F. Lo Verso. This work was supported by the Deutsche Forschungsgemeinschaft in the framework of the Transregio-SFB TR6. We acknowledge also support from SOFT COMP: NoE SoftComp, Contract No. NMP3-CT- 2004-502235. E.Z. acknowledges support from MIUR-Prin and MCRTN-CT2003-504712. MIUR
[1] C. N. Likos, H. Löwen, M. Watzlawek, B. Abbas, O. Jucknischke, J. Allgaier, and D. Richter, Phys. Rev. Lett. 80, 4450 (1998).

[2] G. S. Grest, L. Fetters, J. Huang, and D. Richter, Adv. Chem. Phys. 94, 67 (1996).

[3] J. Stellbrink, J. Allgaier, M. Monkenbusch, D. Richter, A. Lang, C. N. Likos, M. Watzlawek, H. Löwen, G. Ehlers, and P. Schleger, Prog. Colloid Polym. Sci. 115, 88 (2000).

[4] O. Juckniscke, Ph.D. thesis, Universität Münster, 1995 (unpublished).

[5] M. Watzlawek, C. N. Likos, and H. Löwen, Phys. Rev. Lett. 82, 5289 (1999).

[6] G. Foffi, F. Sciortino, P. Tartaglia, E. Zaccarelli, F. Lo Verso, L. Reatto, K. A. Dawson, and C. N. Likos, Phys. Rev. Lett. 90, 238301 (2003).

[7] E. Stiakakis, D. Vlassopoulos, C. N. Likos, J. Roovers, and G. Meier, Phys. Rev. Lett. 89, 208302 (2002).

[8] K. Ishizu, T. Ono, and S. Uchida, J. Colloid Interface Sci. 192, 189 (1997).

[9] D. Richter, O. Jucknischke, L. Willner, L. J. Fetters, M. Lin, J. S. Huang, C. Toporovski, and L. L. Zhou, J. Phys. IV 3, 3 (1993).

[10] J. Roovers, Macromolecules 27, 5359 (1994).

[11] I. W. Hamley, The Physics of Block Copolymers (Oxford University Press, New York, 1998).

[12] A. Halperin, Macromolecules 20, 2943 (1987).

[13] Y. Séréro, R. Aznar, G. Porte, J.-F. Berret, D. Calvet, A. Collet, and M. Viguier, Phys. Rev. Lett. 81, 5584 (1998).

[14] E. Beaudoin, O. Borisov, A. Lapp, L. Billon, R. C. Hiorns, and J. Francois, Macromolecules 35, 7436 (2002).

[15] F. Lafleche, D. Durand, and T. Nicolai, Macromolecules 36, 1331 (2003).

[16] L. Willner, A. Poppe, J. Allgaier, J. Monkenbusch, P. Lindner, and D. Richter, Europhys. Lett. 51, 628 (2000).

[17] R. Lund, L. Willner, J. Stellbrink, A. Radulescu, and D. Richter, Macromolecules 37, 9984 (2004).

[18] G. A. McConnell, A. P. Gast, J. S. Huang, and S. D. Smith, Phys. Rev. Lett. 71, 2102 (1993).

[19] J. Bang, T. P. Lodge, X. Wang, K. L. Brinker, and W. R. Burghardt, Phys. Rev. Lett. 89, 215505 (2002).

[20] T. P. Lodge, J. Bang, M. J. Park, and K. Char, Phys. Rev. Lett. 92, 145501 (2004).

[21] I. W. Hamley, J. A. Pople, and O. Diat, Colloid Polym. Sci. 276, 446 (1998).

[22] M. Laurati, J. Stellbrink, R. Lund, L. Willner, D. Richter, and E. Zaccarelli, Phys. Rev. Lett. 94, 195504 (2005).

[23] A. Poppe, L. Willner, J. Allgaier, J. Stellbrink, and D. Richter, Macromolecules 30, 7462 (1997).

[24] R. Lund, Ph.D. thesis, Universität Münster, 2004 (unpublished).

[25] R. Lund, L. Willner, J. Stellbrink, P. Lindner, and D. Richter,
Phys. Rev. Lett. 96, 068302 (2006).

[26] R. Lund, L. Willner, D. Richter, and E. Dormidontova, Macromolecules 39, 4566 (2006).

[27] G. D. Smith, D. Y. Yoon, R. L. Jaffa, R. H. Colby, R. Krishnamoorti, and L. J. Fetters, Macromolecules 29, 7602 (1996).

[28] M. Laurati, Ph.D. thesis, Universität Münster, 2005 (unpublished).

[29] P. Lindner and Th. Zemb, Neutrons, X-Rays and Light: Scattering Methods Applied to Soft Condensed Matter (NorthHolland, Amsterdam, 2002).

[30] M. Kotlarchyk and S. H. Chen, J. Chem. Phys. 79, 2461 (1983).

[31] J. S. Pedersen, J. Chem. Phys. 114, 2839 (2001).

[32] J. S. Pedersen and C. Svaneborg, Curr. Opin. Colloid Interface Sci. 7, 158 (2002).

[33] V. Castelletto and I. W. Hamley, Curr. Opin. Colloid Interface Sci. 7, 167 (2002).

[34] C. Svaneborg and J. S. Pedersen, Macromolecules 35, 1028 (2002)

[35] S. Förster and C. Burger, Macromolecules 31, 879 (1998).

[36] G. Beaucage, J. Appl. Crystallogr. 28, 717 (1995).

[37] F. J. Rogers and D. A. Young, Phys. Rev. A 30, 999 (1984).

[38] M. Watzlawek, H. Löwen, and C. N. Likos, J. Phys.: Condens. Matter 10, 8189 (1998).

[39] F. Lo Verso, M. Tau, and L. Reatto, J. Phys.: Condens. Matter 15, 1505 (2003).

[40] E. Zaccarelli et al., Phys. Rev. Lett. 95, 268301 (2005).

[41] C. Mayer, E. Stiakakis, E. Zaccarelli, C. N. Likos, F. Sciortino, H. Löwen, P. Tartaglia, and D. Vlassopoulos, Rheol. Acta 46, 611 (2007).

[42] A. Lang, G. Kahl, C. N. Likos, H. Löwen, and M. Watzlawek, J. Phys.: Condens. Matter 11, 10143 (1999).

[43] P. Kumar, S. V. Buldyrev, F. Sciortino, E. Zaccarelli, and H. E. Stanley, Phys. Rev. E 72, 021501 (2005).

[44] C. Sommer, J. S. Pedersen, and P. C. Stein, J. Phys. Chem. B 108, 6242 (2004).

[45] B. Hammouda, D. Ho, and S. Kline, Macromolecules 35, 8578 (2002).

[46] J. S. Pedersen, D. Posselt, and K. Mortensen, J. Appl. Crystallogr. 23, 321 (1990).

[47] J. S. Higgins and H. C. Benoit, Polymers and Neutron Scattering (Oxford University Press, New York, 1994).

[48] L. Willner, O. Jucknischke, D. Richter, J. Roovers, L. L. Zhou, P. Toporowski, L. Fetters, J. Huang, M. Lin, and N. Hadjichristidis, Macromolecules 27, 3821 (1994).

[49] D. Richter, B. Farago, J. S. Huang, L. J. Fetters, and B. Ewen, Macromolecules 22, 468 (1989).

[50] D. Richter, B. Farago, L. J. Fetters, J. S. Huang, and B. Ewen, Macromolecules 23, 1845 (1990).

[51] H. Benoit, J. Polym. Sci. 11, 507 (1953).

[52] J. Stellbrink, J. Allgaier, and D. Richter, Phys. Rev. E 56, 
R3772 (1997).

[53] M. Kapnistos, D. Vlassopoulos, G. Fytas, K. Mortensen, G. Fleischer, and J. Roovers, Phys. Rev. Lett. 85, 4072 (2000).

[54] F. Lo Verso, L. Reatto, G. Foffi, P. Tartaglia, and K. A. Dawson, Phys. Rev. E 70, 061409 (2004).

[55] C. Branca, A. Faraone, G. Maisano, S. Magazú, P. Migliardo, A. Triolo, R. Triolo, and V. Villari, Macromolecules 37, 7747 (1999).

[56] B. Hammouda, D. Ho, and S. Kline, Macromolecules 37, 6932 (2004).

[57] E. Dormidontova, Macromolecules 37, 7747 (2004).

[58] M. Daoud and J. P. Cotton, J. Phys. (Paris) 43, 531 (1982).

[59] J. P. Hansen and L. Verlet, Phys. Rev. 184, 151 (1969).
[60] C. Kittel, Introduction to Solid State Physics (Wiley, New York, 1996).

[61] P. Ziherl and R. D. Kamien, Phys. Rev. Lett. 85, 3528 (2000). [62] J. Bang and T. P. Lodge, Phys. Rev. Lett. 93, 245701 (2004).

[63] T. Nicolai, F. Lafleche, and A. Gibaud, Macromolecules 37, 8066 (2004).

[64] M. Watzlawek, Ph.D. thesis, Universität Düsseldorf, 1999 (unpublished).

[65] W. Ostwald, Z. Phys. Chem., Stoechiom. Verwandtschaftsl. 22, 289 (1897).

[66] F. Sciortino, S. Mossa, E. Zaccarelli, and P. Tartaglia, Phys. Rev. Lett. 93, 055701 (2004). 